\section{Stationäre Phase}

T. Arndt

Bioscientia Institut für Medizinische Diagnostik GmbH, Ingelheim, Deutschland

Synonym(e) Festphase; Packungsmaterial; Sorbens; Trennflüssigkeit

Englischer Begriff stationary phase

Definition Neben der mobilen Phase eine der beiden Phasen, aus denen das chromatographische oder elektrophoretische System besteht ( $\triangleright$ Chromatographie, $\triangleright$ Elektrophorese). Die stationäre Phase ist auf einer planaren Fläche ( $\triangleright$ Dünnschichtchromatographie, $\triangleright$ Flachbett-Elektrophorese) oder in einer Säule (Säulenchromatographie) fixiert. Sie kann aus einem Feststoff (Sorbens), einer Flüssigkeit (Trennflüssigkeit) oder einem Gel bestehen.

Beschreibung Die Trennflüssigkeit ist auf einen Festkörper (Träger) aufgezogen, der auch am Trennprozess beteiligt sein kann.

Die am häufigsten eingesetzten festen Packungsmaterialien sind kugelförmige, poröse anorganische Oxide (z. B. Kieselgel, $\mathrm{Al}_{2} \mathrm{O}_{3}$ ) oder organische Polymere ( $\mathrm{z}$. B. vernetzte Agarose, Copolymere von Styrol-Divinylbenzol, Methylme- thacrylate) oder eine Kombination aus beiden (Verbundpackungsmaterialien). Die Partikeldurchmesser betragen zumeist $3-10 \mu \mathrm{m}$ für analytische und $10-100 \mu \mathrm{m}$ für präparative Anwendungen.

Durch Modifikation der Porengröße und Oberfläche der Packungsmaterialien lässt sich eine enorme Vielzahl von stationären Phasen mit speziellen Trenneigenschaften herstellen. Ein wichtiges Unterscheidungsmerkmal ist die Hydrophobizität der stationären Phase. Eine polare stationäre Phase hat funktionelle Gruppen auf der Sorbensoberfläche. Diese Materialien werden als Normalphasen bezeichnet und die mit ihnen durchgeführte Chromatographie als NormalphasenChromatographie. Durch chemische Modifikation der polaren Gruppen, z. B. durch Bindung von Alkylgruppen mit 8 oder 18 C-Atomen, erhält man unpolare Oberflächen. Man bezeichnet diese stationären Phasen als Umkehrphasen (RP: „reversed phase") und die mit ihnen durchgeführte Chromatographie als Umkehrphasen-Chromatographie (,reversedphase chromatography“").

\section{Literatur}

Ettre LS (1993) Nomenclature for chromatography. Pure Appl Chem 65:819-872

Unger KK (Hrsg) (1989) Handbuch der HPLC. Teil 1 Leitfaden für Anfänger und Praktiker. GIT Verlag, Darmstadt 